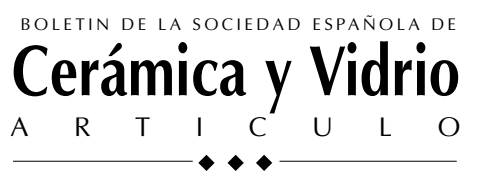

\title{
Situación de la industria argentina de la cerámica, del vidrio y del refractario en el 2000
}

\author{
R. HEVIA \\ INTEMIN-SEGEMAR. Buenos Aires. Argentina
}

La globalización de la política económica actual impone la búsqueda de la excelencia basada en la competitividad.La misma está asociada a las economías de escala como alternativa eficaz para reducir los costos operativos y a la abolición de proteccionismos , que en la Argentina se cumplen rigurosamente. Los efectos de la política se hacen sentir en las distintas áreas de producción de materiales, que de acuerdo con las posibilidades de cada tipo de industria buscan reacomodarse.

En este trabajo se analiza la situación de la industria cerámica en base a los índices de evolución de la construcción en los últimos años, dado que aproximadamente el 90 - $95 \%$ de la producción cerámica argentina está vinculada con ella.También se utilizó la información procedente de las empresas más relevantes y de las cámaras que las nuclean.En el caso de la producción de refractarios y vidrios, sólo se consideró la información proveniente de las empresas.

La producción cerámica se clasificó de acuerdo con los siguientes submercados : cerámica roja , vajilla, pisos y revestimientos y artículos sanitarios. El análisis de las tres industrias citadas incluye la caracterización de la situación actual de cada una de ellas y permite vislumbrar las tendencias predominantes en cada caso.

Palabras clave: cerámica roja, vajilla, revestimientos cerámicos, refractarios, vidrios, industria argentina

\section{Present status of the ceramic glass and refractory industries in Argentina}

The globalization of the present economic policy imposes the search of the excellence based on the competition.The same is associate to the economies of scale like effective alternative to reduce the operative costs and to the abolition of protectionisms, that in Argentina are rigorously fulfilled. The effects of the policy are made feel in the different areas from production of materials, that in agreement with the possibilities of each type of industry they look for to adapt itself. In this work is analyzed the situation of the ceramic industry on the basis of the index of evolution of the construction in the last years, since approximately the $90-95 \%$ of the argentine ceramic production is linked to it.Also was used the information coming from the most excellent companies and of the associations that group them.In the case of the production of refractories and glasses, the originating information of the companies was only considered. The ceramic production was classified in agreement with the following submarkets: red ceramics, tableware, floors and coatings and sanitary ware. The analysis of the three mentioned industries includes the characterization of the present situation of each of them and allows to enhance the predominant tendencies in each case.

Keywords: red ceramic, tiles, tableware, sanitary, refractories, glass, Argentinian industry

\section{INTRODUCCIÓN}

La situación actual de la industria cerámica, del vidrio , del refractario y de la industria en general, está atada a los procesos económicos que se han desarrollado en nuestro país en los últimos 16 años de estabilidad política. Primero fue una etapa de gran inflación y luego siguieron 10 años donde se impuso una política neoliberal, comúnmente llamada "globalización". En esta última etapa se implementó la ley de convertibilidad, que por un lado actuó como un factor estabilizante de la economía, pero a la vez limitante de ajustes necesarios en ciertos momentos.

En un mundo globalizado donde se impone la competitividad empresarial en base a economías de escala y a un fuerte y sostenido desarrollo tecnológico, sumado a la abolición de proteccionismos, nuestra industria ha debido reacomodarse a estas condiciones y el resultado de esa evolución se analizará a continuación.

Considerando que más del 90\% de la producción de la industria cerámica está involucrada con la industria de la construc- ción, se exponen a continuación algunos índices económicos vinculados a la evolución de la misma en los últimos años.

Como fuente de referencia, se tomaron índices de factores económicos como: INDEC, BCRA y de la Dirección de Estadísticas del Gobierno de la Ciudad de Buenos Aires, como así también de Cámaras Sectoriales.

En la década de los 80 la industria de la construcción tuvo un largo período de recesión, su participación en el PBI pasó de $7.6 \%$ en 1981, a un piso de $4.5 \%$ en 1990, recuperándose en el período 1997-1999, que llegó al 6\% (figura 1).

En la figura 2, se muestra la evolución de las ventas de cemento en los últimos 20 años y se puede observar que la expansión lograda en 1997, solamente fue superada en 1980.

Se percibe una tendencia negativa hasta el año 90, tendencia que tiende a revertirse paulatinamente en los últimos años.

Existen diferentes estudios que permiten estimar que el consumo de cerámica roja es aproximadamente un 35\% de las toneladas de cemento despachadas para las obras privadas. 


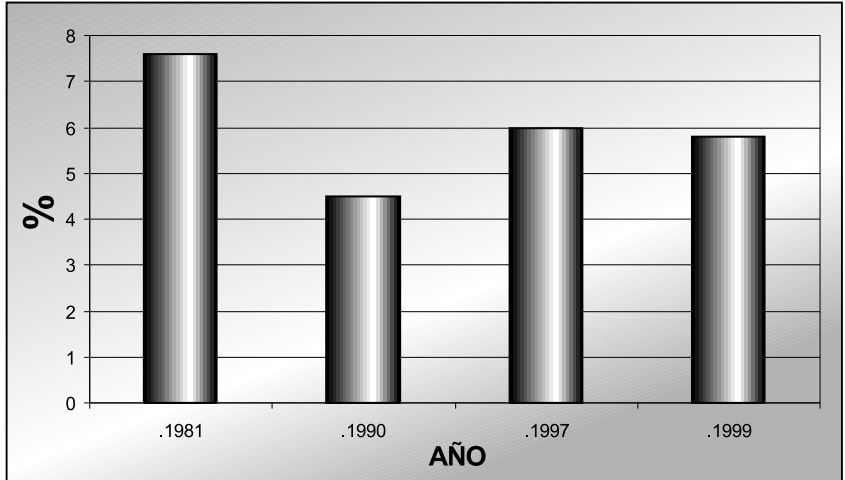

Figura 1. Participación de la construcción en el PIB.

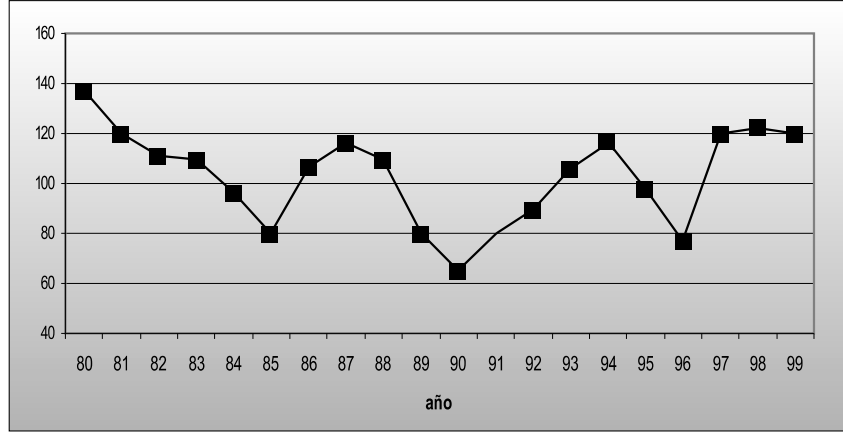

Figura 2. Ventas de cemento.

Del análisis de estadísticas donde se analiza la evolución del número de permisos de construcción anuales, estos se han mantenido constantes dentro de una franja que oscila entre los $65.000-70.000$ permisos. Sin embargo los metros cuadrados que estos permisos implican, han crecido en el último decenio de 10 a 18 millones de metros cuadrados.

Con esta información se puede estimar que ha prevalecido la construcción de grandes obras, con grandes superficies: hipermercados, centros comerciales, hoteles y edificios en propiedad horizontal

Sin embargo, el déficit habitacional sigue siendo muy elevado, estimado por la Secretaría de Desarrollo Social en tres millones de viviendas y es precisamente en el área de vivienda, donde el consumo de productos cerámicos por metro cuadrado de construcción es más importante.

Esta situación genera una importante expectativa para todo el sector cerámico, que mayoritariamente está vinculado a la industria de la construcción, razón por la cual ha estado preparándose tecnológicamente para afrontar con responsabilidad un fuerte requerimiento del mercado.

\section{SITUACIÓN GENERAL DE LA INDUSTRIA CERÁMICA}

En los últimos años, los índices han sido promisorios para la industria cerámica en general. Se puede decir que hubo un crecimiento de consumo interesante que favoreció el crecimiento de varias industrias, con excepción de las de vajilla y porcelana eléctrica.

Otros factores positivos como la baja de los insumos permitió soportar una baja de los precios de venta, que fue general para todos los submercados.
El éxito de cada empresa para afrontar la baja en los precios de venta está relacionada con su capacidad financiera y con la excelencia de su tecnología.

Una constante ha sido que las empresas con éxito fueron las tecnológicamente mejor dotadas y que las que debieron cerrar o cambiar sus paquetes accionarios, fueron aquellas donde su tecnología era inferior o menos productiva y por lo tanto carecían de competitividad.

En la tabla siguiente se expone la variación de la producción durante los últimos 20 años y la capacidad instalada aprovechada en 1999.

TABLA 1. EVOLUCiÓN DE LA PRODUCCIÓN.

\begin{tabular}{|c|c|c|c|c|c|c|c|}
\hline $\begin{array}{c}\text { SUBMERCADO } \\
\text { CERÁMICO }\end{array}$ & UNIDADES & 1978 & 1988 & 1995 & 1997 & 1999 & $\begin{array}{c}\text { CAPACIDAD } \\
\text { UTILIZADA } \\
1999\end{array}$ \\
\hline $\begin{array}{c}\text { CERÁMICA } \\
\text { ROJA }\end{array}$ & t/año & 1.800 .000 & 1.000 .000 & 1.200 .000 & 1.500 .00 & 1.500 .000 & 70 \\
\hline $\begin{array}{c}\text { PISOS Y } \\
\text { REVESTI- } \\
\text { MIENTOS }\end{array}$ & $\mathrm{m}^{2} /$ año & 17.500 .000 & 35.000 .000 & 50.400 .000 & 56.000 .000 & 56.000 .000 & 75 \\
\hline SANITARIOS & artef./año & 2.600 .000 & 3.000 .000 & 3.260 .000 & 3.300 .000 & 3.450 .000 & 80 \\
\hline VAJILLA & artic//año & 53.000 .000 & 35.900 .000 & 15.000 .000 & 10.000 .000 & 9.000 .000 & 50 \\
\hline
\end{tabular}

Estos números se visualizan mejor en un gráfico de barras (Figura 3), donde se destaca la marcada disparidad de crecimiento de los distintos submercados de la industria cerámica argentina: un crecimiento espectacular en el sector de Pisos y Revestimientos, un desarrollo acorde con el crecimiento del país en general para Sanitarios y Cerámica Roja y la declinación permanente de la vajillería.

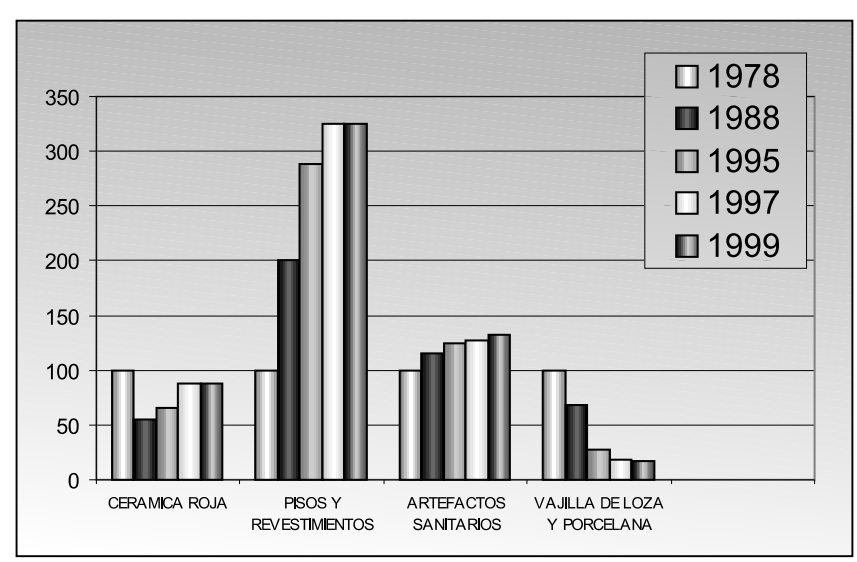

Figura 3. Crecimiento de submercados de la industria cerámica.

\section{SUBMERCADO DE SANITARIOS}

En la figura 4 se representa la distribución productiva de los artículos sanitarios en la Argentina.

FERRUM S.A. es la empresa líder con un 70\% de la producción total.

Producen aproximadamente unas 200.000 piezas por mes entre las dos plantas ubicadas en Avellaneda y Pilar, ambas en la Pcia. de Buenos Aires.

Este Grupo lo integra también la empresa minera Piedra Grande S.A., que en la actualidad es la principal empresa del país proveedora de materias primas para cerámica (caolines lavados, arcillas, cuarzos y feldespatos) 


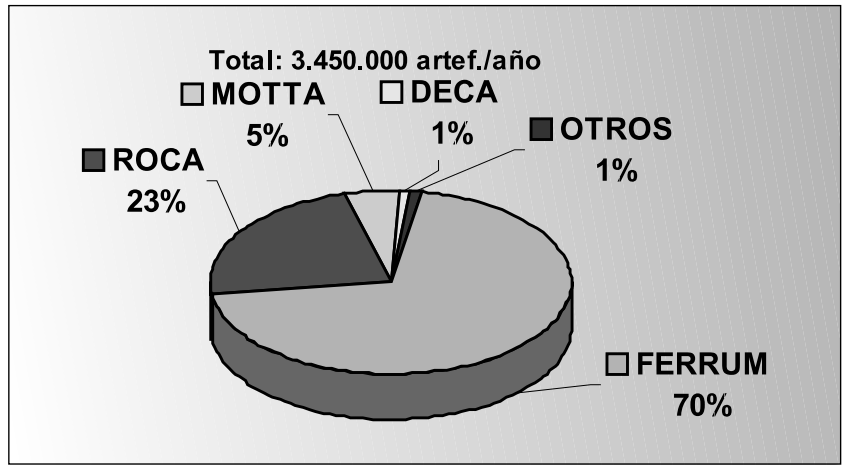

Figura 4. Producción de artículos sanitarios.

MOTTA del GRUPO ZANON, está ubicada en el km 34 del ramal Pilar de la ruta Panamericana, Pcia. de Buenos Aires.

Comprada llave en mano a la empresa SACMI de Italia, comienza su actividad en 1995. La capacidad instalada es de 15.000 piezas por mes y la producción actual es del $80 \%$ aproximadamente.

La incorporación de dos empresas extranjeras, una de origen brasileño y otra de origen español, sumado a las facilidades para la importación originaron, por un lado, aumento de la capacidad instalada, y por otro, una baja importante de los precios de venta.

La empresa brasileña es DECA, una de las divisiones de DURATEX S.A. . Esta empresa es el productor de porcelana y grifería sanitaria más importante de América Latina y se ubica entre los primeros 10 del mundo.

En 1995 Duratex S.A. adquirió el 100\% del paquete accionario de Piazza Hnos, el segundo productor en importancia de la Argentina en el ramo grifería sanitaria, que paso a llamarse Deca-Piazza S.A.

En mayo de 1996 se inició la construcción de la fábrica de porcelana sanitaria, en el Parque Industrial de Pilar, Pcia. de Buenos Aires, a sólo $60 \mathrm{~km}$ de la ciudad de Buenos Aires.

En abril de 1997 comienza a producir la planta industrial.

La empresa española es del Grupo ROCA, líder europeo en productos para el baño, que en 1994 adquirió la mayoría del paquete accionario de Capea, fabricante argentino de porcelana sanitaria e incorporó dos nuevas plantas de producción de $2000 \mathrm{~m}^{2}$ cada una, en el complejo industrial de Lanús, Pcia. de Buenos Aires, donde fabrican unas 800.000 piezas por año, con 300 operarios aproximadamente. Además de una línea económica, fabrican modelos conocidos en Europa como: VICTORIANA, DAMA Y MERIDIAN.

La filosofía de ROCA al igual que FERRUM y DECA, parte del concepto de Coordinación Total del Negocio, basado en la oferta de la gama completa de productos para el baño y la cocina, grifería, etc.

A fines de 1999 puede estimarse que la importación de España, Brasil, Uruguay y Chile llega a los 15.000 artefactos mensuales, para una producción local estimada en 290.000.

Como puede observarse no es muy elevada la importación y esto se debe a la disminución de costos local, que se ha basado en fuertes inversiones en tecnología, racionalización de personas y disminución de gastos no productivos y optimizaron sistemas de combustión.

El desafío que representan las nuevas reglas de juego ha sido asimilado y superado por la industria de sanitarios local y es lógico esperar que su actividad siga un ritmo ascendente.

\section{SUBMERCADO DE LA CERAMICA ROJA}

Haremos solo referencia a los productos más representativos, o sea los extrudados (ladrillos huecos) y tejas (esmaltadas y sin esmaltar).

El ladrillo común no se lo consideró porque, si bien su volumen de producción se estima como importante, no existen datos precisos sobre el sector.

La evolución de la capacidad instalada en este último decenio ha tenido un crecimiento levemente positivo desde un punto de vista tecnológico.

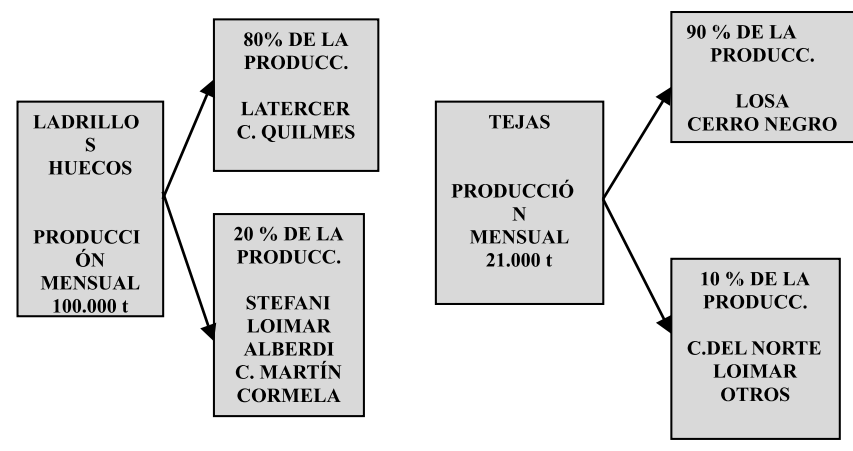

Los niveles de producción de ladrillos huecos alcanzados por las empresas líderes (1.250.000 t/ año), ha permitido que con un grupo pequeño de empresas se abastezca el $90 \%$ del mercado y que su influencia se haga sentir a $30 / 400 \mathrm{~km}$ de las plantas. Entre las empresas más destacadas por su elevado nivel tecnológico se pueden mencionar: Latercer S.A. (Pilar), Cerámica Quilmes S.A.(Quilmes), Loimar S.A. (Tandil), Cerámica Stéfani S.A. (Ruta 3 ,Gran Bs.As.), Cerámica Martín (Isidro Casanova), Cormela (Campana), La Pastoriza (Campana), Cerámica CTIBOR (La Plata), Alberdi (Rosario). Dentro de este grupo, pero con menor capacidad productiva, se puede mencionar a Cerámica Martín, que pertenece a un grupo empresario español, que posee varias fábricas de ladrillos en su país de origen.

El proceso productivo de la cerámica roja de vanguardia se encuentra renovado y en constante mejora: la extrusión, la automatización de los movimientos, la disminución de ciclos y consumos en el secado y cocción están dirigidos no solo a disminuir costos sino, conjuntamente, a mejorar la calidad.

La fabricación de tejas también está concentrada básicamente entre dos empresas líderes que están instaladas en la ciudad de Olavarría, Pcia. de Buenos Aires, que son: LOSA y Canteras Cerro Negro S.A.. Entre ambas tienen el $90 \%$ de la producción total, que es aproximadamente de 7.500 .000 piezas / mes.

El $30 \%$ de la producción corresponde a tejas esmaltadas. También se fabrican partidas especiales de piezas gresificadas, para climas frios.

En este sector no hay exportaciones y solamente la producción se limita al mercado interno. La comercialización se efectúa esencialmente de dos formas: directamente a empresas constructoras o a distribuidores mayoristas (corralones).

SUBMERCADO TOTAL DE PISOS Y DE REVESTIMIENTOS

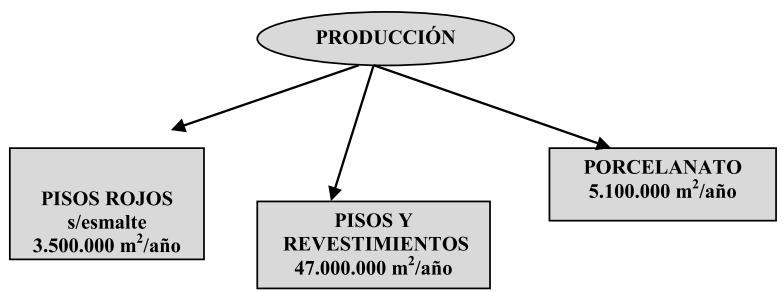


La fabricación de pisos de cerámica roja sin esmalte (producción mensual estimada de 320.000 de metros cuadrados) , que abarca los prensados en medidas pequeñas hasta 20x20cm (Cerámica Cortines y Cerro Negro) y los extrudados (LOSA, Cerámica Alberdi y Loimar) en tamaños mayores, han mantenido su mercado, los primeros con precios bajos y los segundos en base a productos de muy buena presentación.

Una nueva planta con tecnología de punta, perteneciente a LOSA del Grupo Techint se lanza en 1997 al mercado de pisos rústicos por extrusión. La planta produce unos 80.000-100.000 metros cuadrados, con un clásico ciclo de monococción y automatismos similares a los que tienen las plantas más modernas. El producto consiste en placas de $25 \times 25 \mathrm{~cm}$ hasta $60 \times 60 \mathrm{~cm}$, con una amplia gama de accesorios.

La fabricación de porcelanato, continúa creciendo en este sector, ya que a la primer planta de Cerámica ZANON con una producción de 180.000 a 200.000 metros cuadrados mensuales, se le suma ILVA con 150.000 metros cuadrados y Cerámica Lourdes con 100.000 metros cuadrados. Además a estas empresas se les suma Cerámica San Lorenzo que parte de semielaborado importado y pule unos 20.000 metros cuadrados mensuales. Se estima un total mensual de 470.000 metros cuadrados.

El otro submercado de este sector, perteneciente a pisos y revestimientos esmaltados, tiene su progresión del mercado en la siguiente figura.

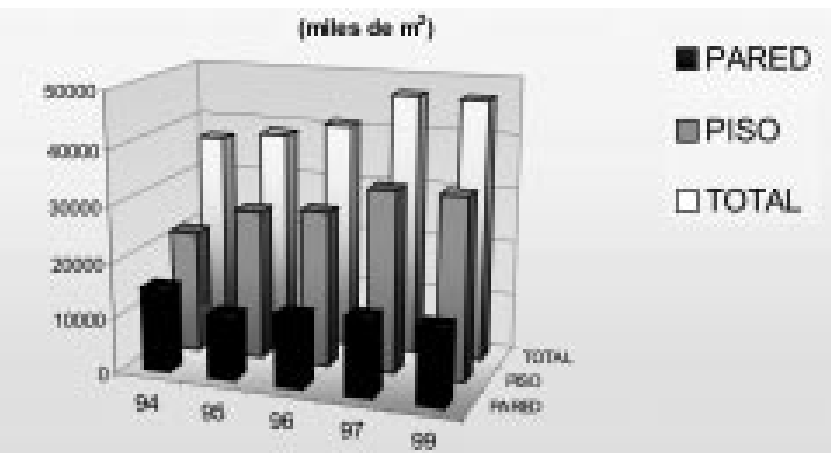

Figura 5. Mercado cerámico. Pisos y revestimientos.

Por la pujanza con que continua creciendo este submercado cerámico desde la aparición de los pisos esmaltados, se puede afirmar con seguridad que es el sector más exitoso de la industria cerámica argentina.

Lo destacable es que el consumidor siga teniendo un hábito de consumo que preferencia el piso esmaltado.

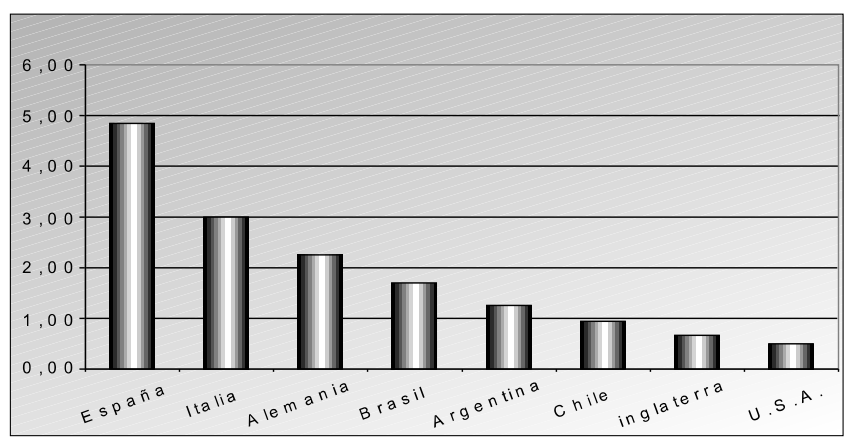

Figura 6. Consumo de pavimentos cerámicos $\left(\mathrm{m}^{2} /\right.$ persona/año).
Estos últimos años se han caracterizado por una fuerte competitividad que determinó el aumento considerable de productos ofrecidos y con precios en descenso.

El aumento de la capacidad instalada se basó en la instalación de una nueva planta, CERAMICA PATAGONIA de Klaukol S.A., hoy perteneciente a Cerámica San Lorenzo y la ampliación de varias otras, llegándose a 1999 a una producción de $47.000 .000 \mathrm{~m}^{2} /$ año, con una utilización del $75 \%$ de la capacidad instalada,

La composición por empresas es la siguiente:

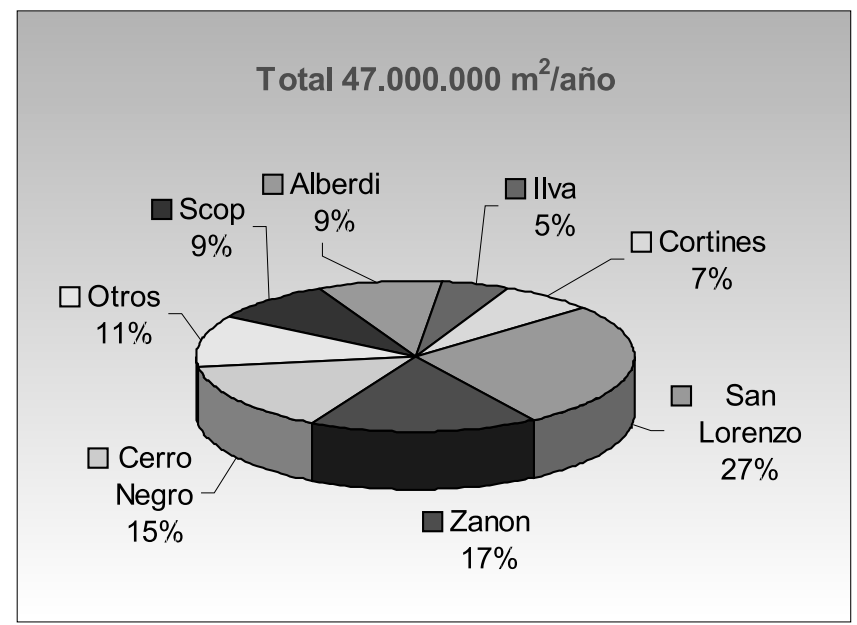

Figura 7. Producción de pisos y revestimientos.

San Lorenzo es un Grupo líder en Argentina, que nació en el año 1952, en la Provincia de Santa Fe, precisamente en la ciudad de San Lorenzo con la fabricación de azulejos.

El Grupo se constituye actualmente con las siguientes empresas:

-Cerámica San Lorenzo (Azul, Pcia. de Buenos Aires) que produce aproximadamente $10.000 .000 \mathrm{~m}^{2}$ anuales .

Cerámica Patagonia (Pto. Madryn, Pcia. de Chubut), tiene un volumen de producción anual de $2.500 .000 \mathrm{~m}^{2}$ en pasta blanca.

-Cerámica SCOP, es una moderna planta ubicada en la provincia de San Juan, con un volumen de producción cercana a los $5.100 .000 \mathrm{~m}^{2}$ anuales

-Cerámica Sassuolo, en la Pcia. de San Luis, con una producción anual estimada en $3.070 .000 \mathrm{~m}^{2} /$ mes

Canteras Cerro Negro S.A. , otra de la empresas importantes de este sector tiene en Olavarría (Pcia. de Buenos Aires) una línea de producción con una capacidad de casi 5.000.000 $\mathrm{m}^{2}$ por año.

El denominador común de las plantas ha sido la renovación tecnológica en la más variada gama de emprendimientos: renovación de hornos de baja productividad y / o alto consumo energético, por hornos de alta producción, inclusive algunos de doble canal que permite cocinar en él, dos tipos diferentes de productos en forma simultánea, nuevas prensa de alto rendimiento, incorporación de robots, decoración con rotativas, todo ello acompañado por racionalización de mano de obra, disminución de personal administrativo y la integración de plantas. Todo esto ha llevado a que los $300 \mathrm{~m}^{2} /$ operario/mes de 1974 y los 3000 pronosticados 
en 1995, han sido superados y hoy podemos hablar de $4000 \mathrm{~m}^{2} /$ operario/mes.

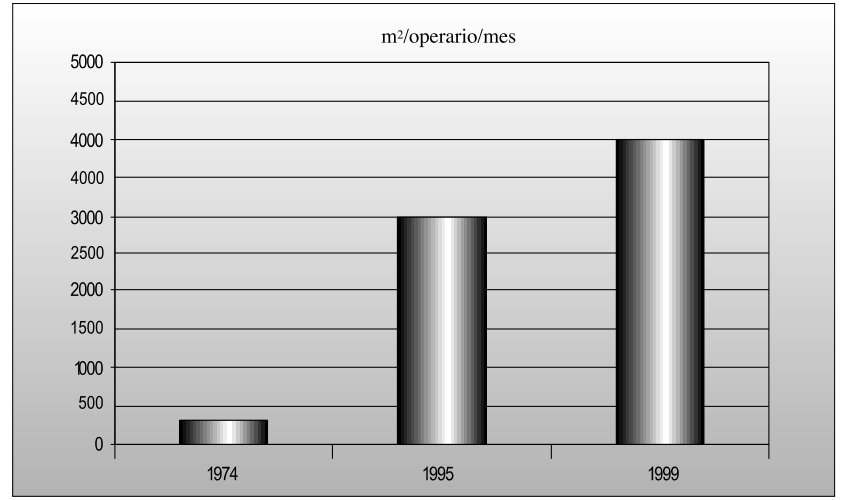

Figura 8. Pisos y revestimientos. Variación de la producción mensual.

Otro factor que es importante señalar es la capacidad que el sector ha demostrado para exportar en mercados exigentes como: Inglaterra, Estados Unidos, Alemania, Suecia y en los países sudamericanos.

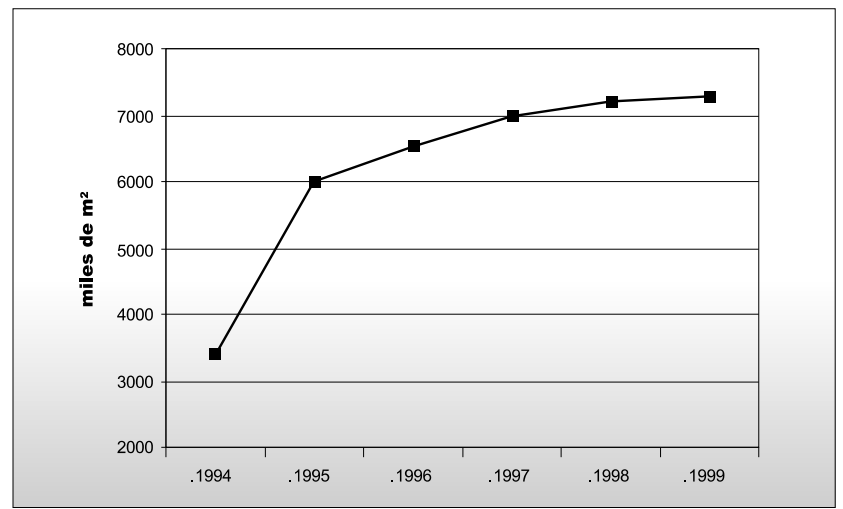

Figura 9. Exportaciones de pavimentos cerámicos.

El balance comercial da un saldo escasamente positivo porque la importación de pisos y revestimientos proveniente principalmente de Brasil, España, Italia, se estima en un 8$10 \%$ de la producción total, es decir 4 y $5.000 .000 \mathrm{~m}^{2} /$ año

\section{SUBMERCADO DE VAJILLA DE LOZA Y PORCELANA}

Este es el sector más afectado por la competencia externa. La mercadería de origen oriental se comercializa a precios inalcanzables para la industria nacional, a pesar que en muchos casos dicha mercadería no es de primera calidad. También se importa vajilla de Uruguay, Brasil y en menor grado de Europa.

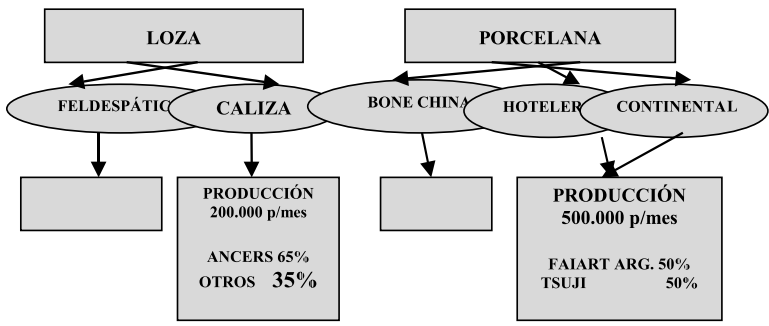

Boletín de la Sociedad Española de Cerámica y Vidrio. Vol. 40 Núm. 1 Enero-Febrero 2001
Hasta fines de la década del 80 Argentina tenía una producción que superaba las 4.000.000 de piezas mensuales de loza feldespática y las 800.000 piezas mensuales de loza caliza.

Actualmente no hay fabricación de loza feldespática y en loza caliza la producción no supera las 200.000 piezas mensuales, quedando solamente Ancers S.A. como empresa líder del sector con un $65 \%$ de la producción total.

De las tres importantes empresas que fabricaban porcelana, actualmente existen Tsuji S.A., ubicada en Burzaco - Gran Buenos Aires y Faiart Argentina S.A. (ex Verbano), ubicada en Capitán Bermudez - Santa Fe . Ambas están produciendo unas 6.000.000 de piezas por año y tiene cada una un $50 \%$ aproximadamente de la producción total.

\section{INDUSTRIA REFRACTARIA}

La falta de inversiones intensivas en tecnología y una real disminución del consumo específico de refractarios, en los últimos años, han llevado a nuestra industria refractaria a una franca declinación.

La industria siderúrgica, que junto a la del cemento consume el $90 \%$ de los refractarios que se fabrican en el país, se provee básicamente en Brasil, aunque los de origen chino van ganando mercado.

La producción anual es de aproximadamente 62.000 t.. La distribución de la misma entre las principales empresas se expone en la Tabla 2.

TABLA 2.

\begin{tabular}{|c|c|c|}
\hline INDUSTRIA & $\begin{array}{c}\text { PRODUCCIÓN } \\
\mathrm{t} / \mathrm{m}\end{array}$ & PRODUCTOS \\
\hline $\begin{array}{c}\text { CERAMICA } \\
\text { AVELLANEDA }\end{array}$ & 800 & SíLICO-ALUMINOSOS \\
\hline RASA & 3.000 & BÁSICOS \\
\hline FARA & 1.500 & SíLICO-ALUMINOSOS \\
\hline CUARSABRAS & 300 & $\begin{array}{c}\text { ESPECIALIDADES (hormigones, } \\
\text { plásticos y morteros) }\end{array}$ \\
\hline OTROS & 400 & SílICO-ALUMINOSOS \\
\hline
\end{tabular}

En 1997 Morgan-Thermal Ceramics adquirió parte del paquete accionario de Carbo San Luis para instalar una planta de fibras cerámicas que se puso en marcha en agosto del 98. Ya se han realizado exportaciones a Chile, Uruguay y EE:UU:

\section{INDUSTRIA DEL VIDRIO}

\subsection{Estructura del sector industrial}

En los últimos diez años la industria vidriera argentina ha presentado una evolución dinámica con períodos de retracción, cierre de fábricas, concentración empresaria, nuevas inversiones, adaptación de desarrollos tecnológicos, sustitución de materiales y otros factores, resultado de los vaivenes de la evolución económica local y de la globalización.

El sector vidriero está agrupado en tres cámaras empresariales: la Cámara Argentina de Fabricantes de Vidrio, la cual nuclea fundamentalmente a los productores de vidrio de todo tipo. La Cámara del Vidrio Plano y sus Manufacturas, que congrega a los grandes distribuidores/mayoristas y/o transformadores de vidrio plano y la Cámara Argentina Minorista del Vidrio Plano, la cual agrupa a las empresas minoristas que comercializan e instalan el vidrio plano. 


\subsection{Procesos y productos}

El porcentaje de los distintos sectores de la industria del vidrio argentina en base a la capacidad instalada es la siguiente: (datos propios del año 98/99)

Vidrio para envases: $70 \%$

Vidrio plano: $21 \%$

Vajilla: $6 \%$

Varios: $3 \%$

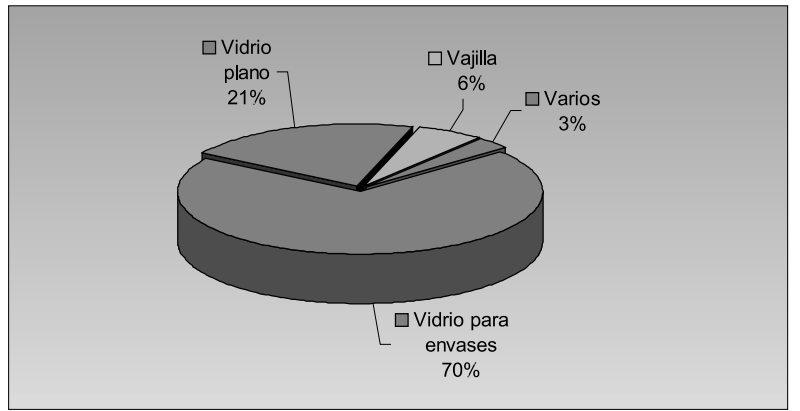

Figura 10. Proporción sectorial según la capacidad instalada.

\subsubsection{ENVASES}

Existen tres empresas fabricantes con ocho plantas industriales, están ubicadas en el gran Buenos Aires y en la zona de Cuyo. La capacidad instalada total (estimada) es de 1.000.000 t/ año y su facturación estimada alcanzó los 320 M USD.

El aprovechamiento actual de la capacidad instalada es de aproximadamente un $70 \%$.

TABLA 3.

\begin{tabular}{|l|l|l|l|}
\hline GRUPO EMPRESARIO & EMPRESA & & \% DEL MERCADO \\
\hline CATTORINI HNOS & $\begin{array}{l}\text { Cattorini: } \\
\text { 1) La Ideal } \\
\text { 2) Pasco } \\
\text { 3) San Juan }\end{array}$ & $\begin{array}{l}\text { Quilmes } \\
\text { Quilmes } \\
\text { San Juan }\end{array}$ \\
\cline { 2 - 3 } & Rygolleau & \multirow{4}{*}{ Berazategui } \\
\cline { 2 - 3 } & N. Cristal. Avellaneda & Lanus & \multirow{2}{*}{$\mathbf{2 0}$} \\
\cline { 2 - 3 } $\begin{array}{l}\text { VITRA ARG. } \\
\text { (Ex. Crist. De Cuyo) }\end{array}$ & & Mendoza & \\
\hline RAYEN CURÁ S.A. & & Rosario & $\mathbf{1 0}$ \\
\hline
\end{tabular}

Es el sector más fuerte en ventas, en volúmenes y en competencia. Hasta hace un par de años existían seis empresas fabricantes de envases con una estructura de capital $100 \%$ nacional. Actualmente, una de las empresas de capital nacional (Rayén Curá) ha sido adquirida por el grupo Saint Gobain y depende directamente de Cristalería Española, que pertenece a dicho grupo. Además, se ha producido una concentración tal que la fabricación de envases ha quedado en manos de tres grupos empresariales.

Distribución del consumo por producto (1998):

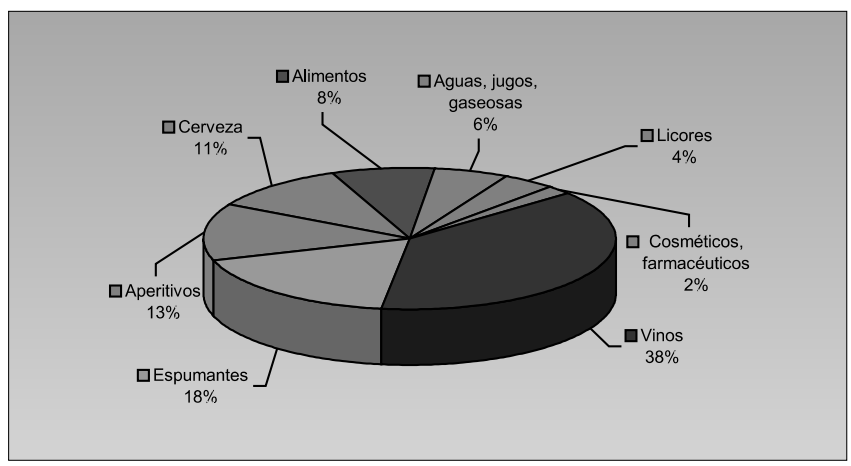

Figura 11. Envases.
La balanza comercial del sector envases durante el año 1999 se presenta en la siguiente tabla: (Indec, Comercio exterior, exportaciones-importaciones 1999) El consumo de envases per cápita para 1999 fue de 13,6 kg por habitante.

TABLA 4.

\begin{tabular}{|c|c|}
\hline Importación (CIF USD) & Exportación (FOB USD) \\
\hline $\mathbf{1 5 . 4 7 0 . 5 3 6}$ & $\mathbf{8 . 5 0 7 . 2 4 0}$ \\
\hline
\end{tabular}

\subsubsection{VAJILLA}

Se registran actualmente dos fabricantes de vajilla (Rigolleau S.A. y Cristalux S.A.) de vidrio soda cal y opal, que producen: platos, tazas, vasos y copas, tanto de vidrio recocido como templado, incoloro y de color (ámbar, gris, bronce, azul) La capacidad instalada es del orden de las 55.000 t/ año.
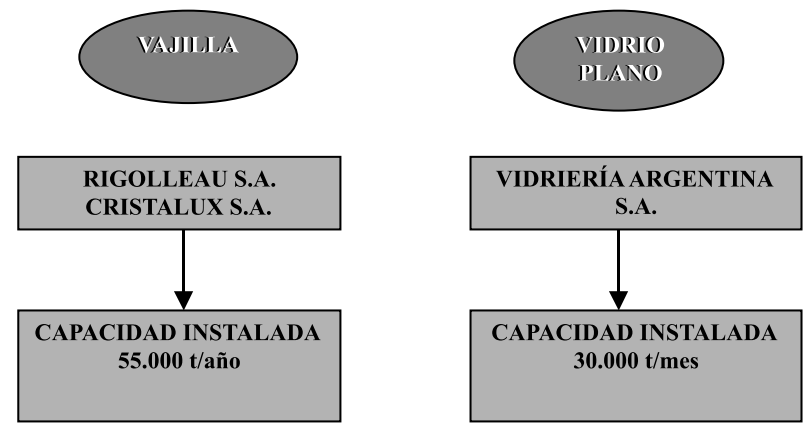

También existen varios pequeños fabricantes de artículos de bazar y de adorno de vidrio soda cal. Se trata de empresas con producción manual o semiautomática.

Se ha dejado de fabricar vajilla térmica de vidrio borosilicato. La única fabrica que quedaba en el país dejó de producir hace aproximadamente un año.

\subsubsection{FIBRA DE VIDRIO PARA AISLACIÓN TÉRMICA Y ACÚSTICA}

Existen dos fabricantes de este producto, una vinculada al grupo Saint Gobain y otra de capitales nacionales.

\subsubsection{Vidrio Plano:}

Existe en nuestro país un solo fabricante de vidrio plano que es Vidriería Argentina S.A., cuya estructura de capital es 51\% del grupo Pilkington y 49\% del grupo Saint Gobain. La planta industrial está ubicada en Llavallol, a unos $27 \mathrm{~km}$ de la ciudad de Buenos Aires: Posee un horno de vidrio plano flotado con una capacidad de aprox. $3500 \mathrm{t} /$ semana.

\subsubsection{TRANSFORMADORES DE VIDRIO PLANO}

Existen una docena de empresas medianas y pequeñas que se dedican a la transformación de vidrio plano ya sea para la industria automotriz como para la construcción. Los productos ofrecidos por estas empresas van desde vidrios templados y laminados hasta doble vidriado hermético y espejos. En el rubro de DVH se nota un crecimiento sustantivo puesto que actualmente hay ocho empresas con líneas de producción de alta tecnología, el uso del DVH se ha incrementado fuertemente en los últimos años especialmente para los edificios de oficinas en zona de alto nivel de ruido. 